\title{
Physics prospects at the HL-LHC with ATLAS
}

\author{
Anna Duncan*广 \\ University of Glasgow \\ E-mail: a.duncan.2@research.gla.ac.uk
}

The High-Luminosity LHC aims to provide a total integrated luminosity of $3000 \mathrm{fb}^{-1}$ from proton-proton collisions at $\sqrt{s}=14 \mathrm{TeV}$ over the course of $\sim 10$ years, reaching instantaneous luminosities of up to $L=7.5 \times 10^{34} \mathrm{~cm}^{-2} \mathrm{~s}^{-1}$, corresponding to an average of 200 inelastic pp collisions per bunch crossing $(\mu=200)$. The upgraded ATLAS detector and trigger system must be able to cope well with increased occupancies and data rates. The performance of the upgrade has been estimated in full simulation studies, assuming expected HL-LHC conditions and a detector configuration intended to maximise physics performance and discovery potential at the HL-LHC, and is expected to be similar to current performance. Fast simulation studies have been carried out to evaluate the prospects of various benchmark physics analyses to be performed using the upgraded ATLAS detector with the full HL-LHC dataset.

The 15th International Conference on Flavor Physics CP Violation

5-9 June 2017

Prague, Czech Republic

\footnotetext{
* Speaker.

${ }^{\dagger}$ These proceedings include results from four HL-LHC physics prospects studies carried out by members of the ATLAS collaboration and coordinated by the ATLAS Upgrade group.
} 


\section{HL-LHC and the Phase-II ATLAS Upgrade}

The $3000 \mathrm{fb}^{-1}$ total integrated luminosity of proton-proton collisions delivered by the HL-LHC beginning in 2026 will significantly improve physics capabilities. Analyses will be able to probe the multi TeV scale in searches for new heavy particles, make higher precision Standard Model measurements, and set stronger limits on/ have improved sensitivity to rare processes. In order to achieve the target integrated luminosity, the HL-LHC will reach instantaneous luminosities of up to $L=7.5 \times 10^{34} \mathrm{~cm}^{-2} \mathrm{~s}^{-1}$, giving rise to a very challenging environment. An average of 200 inelastic proton-proton interactions per bunch crossing $(\langle\mu\rangle=200)$ is expected. The higher occupancy will degrade jet reconstruction, and event reconstruction in general, and increased data rates will cause a non-linear increase of trigger rates. Superior detector performance in pileup rejection, jet reconstruction and flavour tagging will be essential for maintaining physics performance with ATLAS under HL-LHC conditions.

The phase-II ATLAS upgrade will involve replacements and modifications of the existing ATLAS subdetectors. The current inner detector will be replaced with an all silicon tracking system: a pixel detector surrounded by a large-area strip tracking detector. Its will have higher granularity to reduce occupancy, and extended pseudorapidity coverage: $(|\eta|<4.0)$. A new trigger/ DAQ architecture will be implemented to cope with increased data rates.

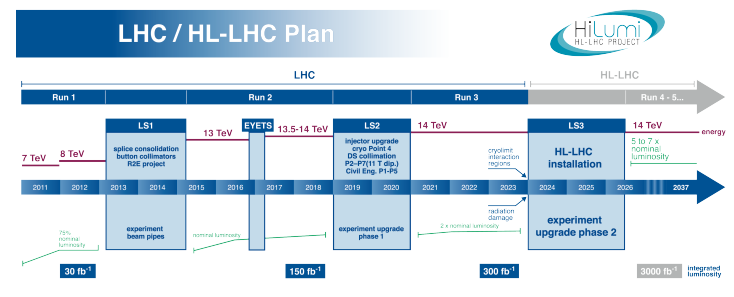

Figure 1: LHC Timeline

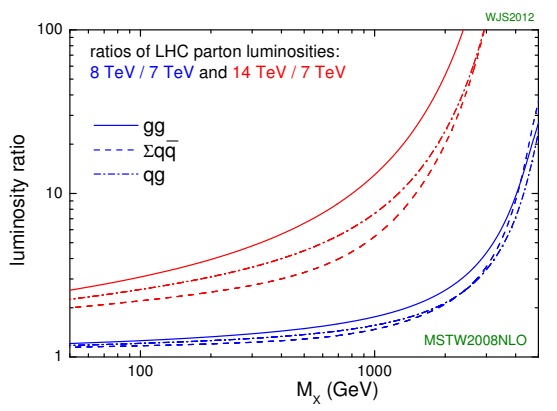

Figure 2: LHC parton luminosity ratios [2]

\section{Physics Prospects Studies and Detector Effects}

Fast simulation studies have been carried out to evaluate the prospects of benchmark analyses at HL-LHC. The expected performance of the phase-II upgrade has been estimated for physics objects and trigger/ DAQ with a fully simulated detector and assuming pile-up of $\langle\mu\rangle=200$ [1]. Parameterised estimates of performance were derived from the full simulation studies and implemented in some of the prospects studies, allowing "smeared truth" simulations of analyses to be carried out. Other studies used extrapolations from Run 1 and Run 2 as performance estimates.

The following prospects studies are based on analyses which will be interesting to see at the HL-LHC due to their potential sensitivity to the increased energy and statistics; a search for a heavy particle, precision measurements in the Higgs sector and a search for a rare process. 


\section{Search for $t \bar{t}$ Resonances}

Several theories of physics beyond the Standard Model predict new particles with masses in the $\mathrm{TeV}$ scale that decay to a top - anti top quark $(t \bar{t})$ pair. A search for such particles, or resonances, is a benchmark analysis for evaluating physics prospects at the HL-LHC. The $14 \mathrm{TeV}$ collision energy will improve the mass reach of the search and the increase in statistics of high $p_{T}$ events will tighten the upper limits that, in the absence of signal, can be set on the cross sections of hypothesised heavy particles. A $t \bar{t}$ resonance would cause a local excess or deficit in the $t \bar{t}$ mass spectrum as predicted by the Standard Model. A search for such a deviation is performed on $t \bar{t}$ pairs selected from events in Monte Carlo simulations of proton-proton collisions with a centre of mass energy of $14 \mathrm{TeV}$, over a total integrated luminosity of $3000 \mathrm{fb}^{-1}$. The search is performed in the semi-leptonic decay channel: both top quarks decay to a b quark and a $\mathrm{W}$ boson; one $\mathrm{W}$ decays to two quarks and the other decays to a lepton and a neutrino. Detector effects are accounted for using parameterised estimates of the performance of an upgraded ATLAS detector. This search relies on a good reconstruction of boosted objects, and is dependent on upgrade tracking performance in a dense environment for b-tagging and lepton isolation. The $t \bar{t}$ invariant mass spectrum is examined for local excesses or deficits. A statistical analysis sets expected upper limits on the cross section of a $t \bar{t}$ resonance in a benchmark theoretical model for several signal masses. A systematic uncertainty on the total signal and background yield in each channel is applied: $8.8 \%$ on the resolved signal channel, $18.0 \%$ on the boosted signal channel, $10.8 \%$ on the resolved background channel and $13.4 \%$ on the boosted background channel. The uncertainties are taken from the Run 1 analysis [4] and reflect the average impact of the dominant systematic uncertainties on the yields. The mass reach of a search for a Z' boson in a TopColour model at the HL-LHC is estimated to be $4 \mathrm{TeV}$. This is an increase of $\sim 1 \mathrm{TeV}$ relative to the estimated limit using $300 \mathrm{fb}^{-1}$ that is expected from the LHC prior to the upgrade [3]. The strongest mass limit on this resonance is currently $2.1 \mathrm{TeV}$ [4].

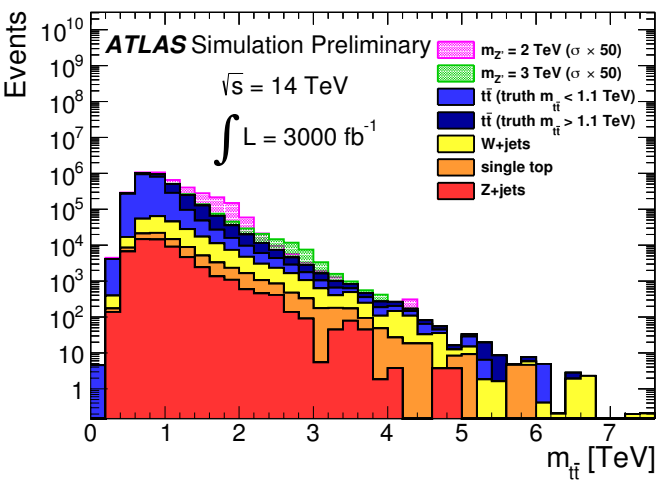

Figure 3: $m_{t \bar{t}}$ spectrum in the boosted electron channel [3].

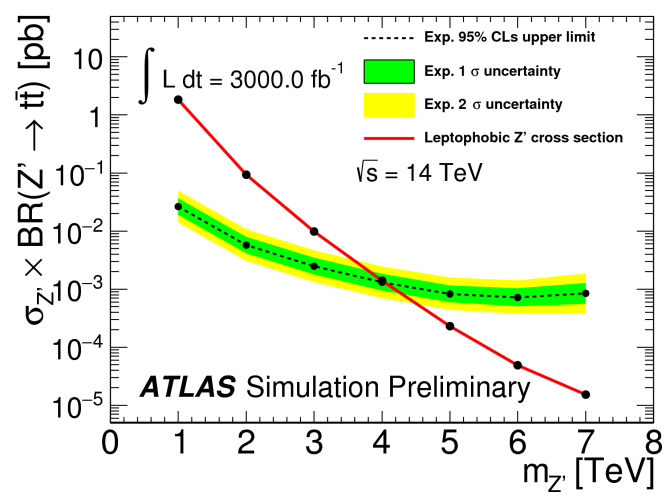

Figure 4: Expected upper limits set on the cross section times branching ratio of a $Z$ ' boson at different mass points [3]. 


\section{Higgs Boson Production via Vector Boson Fusion}

Vector boson fusion (VBF) is the Higgs Boson production mechanism with the second highest cross-section after gluon-gluon fusion (ggF) (Figure 5). In VBF production, the Higgs boson is produced along with two jets in the forward region. The unique signature of two forward jets with large dijet invariant mass means that a good signal to background ratio can be achieved in the event selection. Additionally, the theoretical uncertainty on the VBF Higgs production cross-section is low relative to ggF. Therefore a precision measurement on this process is possible with sufficient statistics. This study [5] estimates the precision with which ATLAS will be able to measure VBF Higgs production at the HL-LHC. The expected precision depends on the inner tracker coverage as this dictates the degree to which the forward jet signature can be exploited. The analysis uses a central jet veto (CJV), where events are rejected if there is a high $p_{T}$ jet inside the rapidity range of the two leading $p_{T}$ jets. Increased tracker acceptance in $|\eta|$ allows for better pileup rejection, leading to fewer signal events being rejected by the CJV. In this measurement, events are selected where the Higgs decays to two $\mathrm{W}$ bosons which in turn each decay to a lepton and a neutrino, so it relies on the performance of lepton and missing transverse energy reconstruction. The analysis requires a b-jet veto to mitigate the $t \bar{t}$ background, and so is also sensitive to b-tagging performance. The quoted precision of the measurement is the uncertainty on the signal strength. Figure 7 shows the expected precision (neglecting theoretical uncertainties on $\mathrm{ggF}$ and VBF production) for three different scenarios of inner tracker $|\eta|$ coverage. The increased tracker coverage allows a precision measurement of VBF Higgs production even in a high pileup environment.
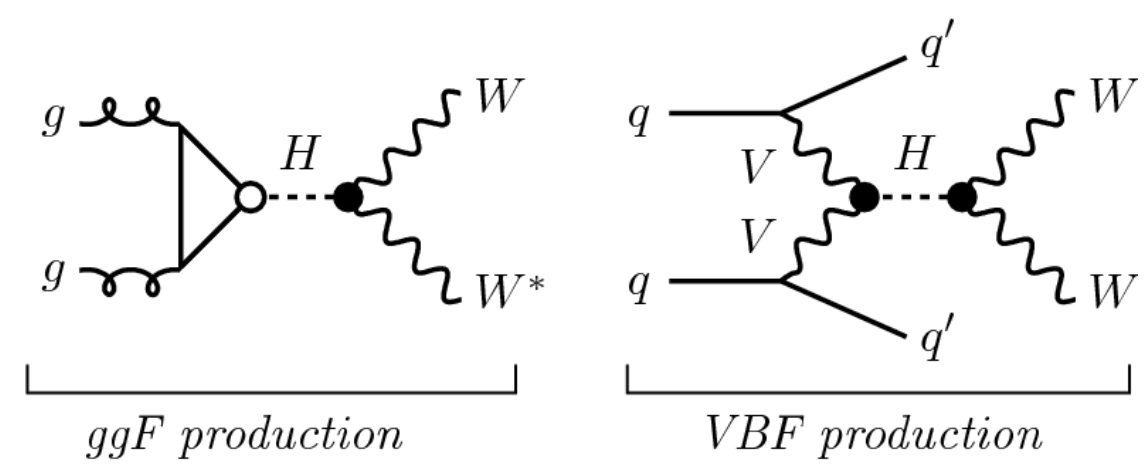

Figure 5: Higgs production mechanisms [5].

\section{Flavour Changing Neutral Current Top Quark Decay}

Flavour changing neutral currents (FCNC) in top quark decays are highly suppressed in the Standard Model with rates $<10^{10}$ and are sensitive to some hypothesised new physical processes; any detection of this decay would be a sign of new physics. This study [6] estimates the expected sensitivity to the FCNC top quark decays $t \rightarrow Z q$ and $t \rightarrow H q$ at HL-LHC. The decay channels considered are $t \bar{t} \rightarrow W(\rightarrow \ell v) b Z(\rightarrow \ell \ell) u / c$ and $t \bar{t} \rightarrow W(\rightarrow \ell v) H(\rightarrow b b) u / c$. Events are selected from Monte Carlo simulations of signal and background, and expected upper 95\% CL limits are set on FCNC top quark branching ratios. This is done for no systematic uncertainties, and for two sets 


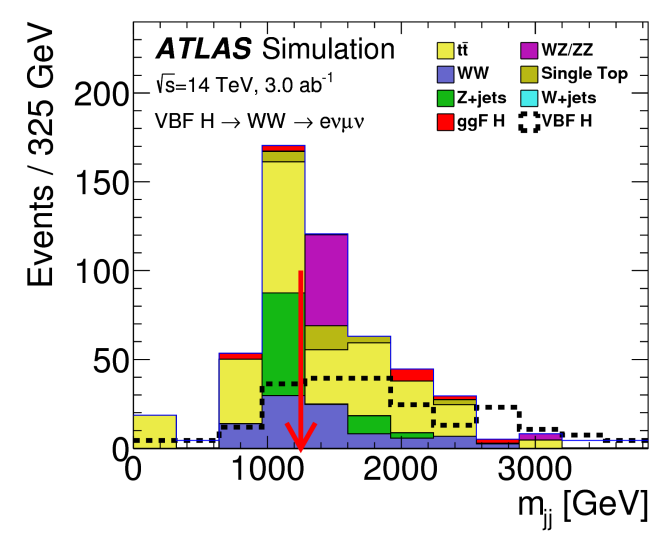

\begin{tabular}{|c|c|}
\hline Tracker Coverage & Expected Precision \\
\hline$|\eta|<4.0$ & $14 \%$ \\
\hline$|\eta|<3.2$ & $20 \%$ \\
\hline$|\eta|<2.7$ & $30 \%$ \\
\hline
\end{tabular}

Figure 7: Expected precision of VBF HH signal measurement [5].

Figure 6: Forward dijet invariant mass spectrum for signal and background events [5].

\begin{tabular}{|c|c|c|}
\hline Uncertainties & $t \rightarrow Z q$ channels & $t \rightarrow H q$ \\
\hline statistics only & $(2.4-5.8) \times 10^{-5}$ & $(0.6-1.2) \times 10^{-4}$ \\
\hline statistics + systematics (A) & $(12-41) \times 10^{-5}$ & $(1.1-2.4) \times 10^{-4}$ \\
\hline statistics + systematics (B) & $(8.3-24) \times 10^{-5}$ & $(1.1-2.4) \times 10^{-4}$ \\
\hline
\end{tabular}

Table 1: Expected upper 95\% CL limits on FCNC top quark branching ratios [6].

of systematic uncertainties: A and B. Systematics A are based on Run 1 estimates where Monte Carlo predictions are compared with data in control regions. Systematics B conservatively account for improvements to dominant theoretical and background normalisation uncertainties which can be achieved with HL-LHC statistics. Detector related systematic uncertainties are considered to be negligible. Table 1 shows the expected upper limits on FCNC top quark decay for each decay channel and systematics scenario. The systematics have a lower impact on $t \rightarrow H q$ sensitivity due to larger expected statistics and multiple fit regions. The strongest observed limits from Run 1 are $\mathscr{B}(t \rightarrow Z q)<50 \times 10^{-5}, \mathscr{B}(t \rightarrow H u)<45 \times 10^{-4}$ and $\mathscr{B}(t \rightarrow H c)<46 \times 10^{-4}$ [7], [8].

\section{Measuring the Higgs Trilinear Self-Coupling through Higgs Pair Production}

One of the primary goals of LHC physics is to establish whether the Higgs mechanism is responsible for electroweak symmetry breaking (EWSB). This requires extensive investigation of Higgs boson properties, including the Higgs trilinear self-coupling, $\lambda_{H H H}$. The processes represented by the diagrams in Figures 8 and 9 interfere destructively, so any deviation in $\lambda_{H H H}$ will modify non-resonant Higgs pair production. The low Standard Model HH production cross section means that it has not yet been observed; the best $95 \%$ CL limit on HH production so far is $\sigma / \sigma_{S M} \leq 29$, obtained from an analysis performed on ATLAS Run 2 data searching in the dominant $H H \rightarrow b b b b$ decay channel. This study [9] extrapolates the ATLAS Run 2 results to estimate the prospects $H H$ production measurement at HL-LHC, which in turn can be used to place limits on $\lambda_{H H H}$. The chosen $H H \rightarrow b b b b$ decay channel means that b-tagging performance of the upgrade is critical to this analysis. It is assumed that the upgrade jet reconstruction and b-tagging performance is the same 
as for the current detector, and that the analysis method remains the same, which a conservative assumption. The $H H \rightarrow b b b b$ search translated to constraints on $\lambda_{H H H}$ by exploiting the fact that $\lambda_{H H H}$ variations change the $H H$ cross section ad the shape of the $m_{H H}$ distribution. The $95 \% \mathrm{CL}$ constraints on $\lambda_{H H H}$ are $-3.5<\lambda_{H H H} / \lambda_{H H H}^{S M}<11$ in the case where systematic uncertainties remain as they were in Run 2 (Figure 10) and $0.2<\lambda_{H H H} / \lambda_{H H H}^{S M}<7.0$ in the case when systematic uncertainties are negligible (Figure 11).

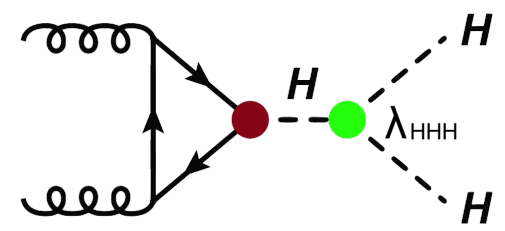

Figure 8: HH production with Higgs selfcoupling vertex [9].

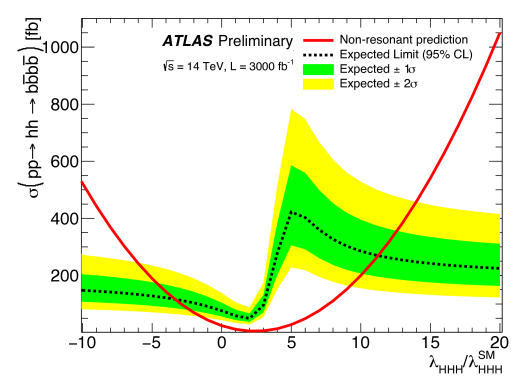

Figure 10: Expected constraints on $\lambda_{H H H}$ with current systematic uncertainties included [9].

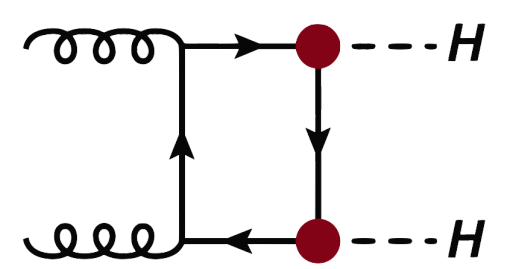

Figure 9: Non-resonant HH production [9].

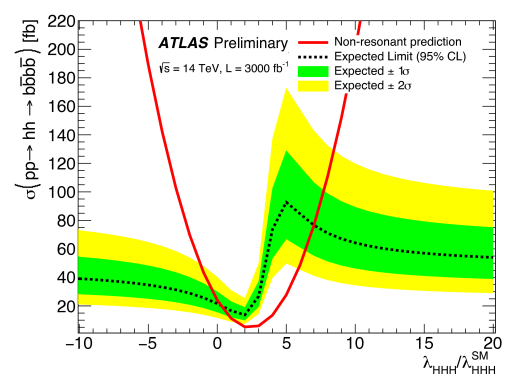

Figure 11: Expected constraints on $\lambda_{H H H}$ with no systematic uncertainties [9].

\section{References}

[1] ATLAS Collaboration. Expected performance for an upgraded ATLAS detector at High-Luminosity LHC. ATLAS-PUB-2015-026, url: https://cds.cern.ch/record/2055248

[2] W.J. Stirling, private communication

[3] ATLAS Collaboration. Study on the prospects of a $t \bar{t}$ resonance search in events with one lepton at a High Luminosity LHC. ATL-PHYS-PUB-2017-002, url: https://cds.cern.ch/record/2243753

[4] ATLAS Collaboration. A search for $t \bar{t}$ resonances using lepton-plus-jets events in proton-proton collisions at $\sqrt{s}=8 \mathrm{TeV}$ with the ATLAS detector. JHEP 08 (2015) 053, arXiv:1505.07018 [hep-ex]

[5] ATLAS Collaboration. Measurement prospects for $V B F H \rightarrow W W * \rightarrow$ ev $\mu v$ production with 3 ab $b^{-1}$ of HL-LHC pp-collisions. ATL-PHYS-PUB-2016-018, url: http://cds.cern.ch/record/2209092

[6] ATLAS Collaboration Expected sensitivity of ATLAS to FCNC top quark decays $t \rightarrow Z q$ and $t \rightarrow H q$ at the High Luminosity LHC. ATL-PHYS-PUB-2016-019, url: https://cds.cern.ch/record/2209126

[7] ATLAS Collaboration. Search for flavour-changing neutral current top quark decays $t \rightarrow H q$ in $p p$ collisions at $\sqrt{s}=8 \mathrm{TeV}$ with the ATLAS detector. JHEP 12 (2015) 061, arXiv:1509.06047 [hep-ex], 
[8] CMS Collaboration. Search for flavor-changing neutral currents in top-quark decays t to Zq in pp collisions at $\sqrt{s}=8 \mathrm{TeV}$ Phys. Rev. Lett. 112 (2014) 171802, arXiv:1312.4194

[9] ATLAS Collaboration Projected sensitivity to non-resonant Higgs boson pair production in the b $\bar{b} b \bar{b}$ final state using proton-proton collisions at HL-LHC with the ATLAS detector ATL-PHYS-PUB-2016-024, url: http://cds.cern.ch/record/2221658/ 